\title{
Epidemiological, Diagnostic, Therapeutic and Evolutionary Profile of Patients with Follicular Lymphoma from 2012 to 2015 in Casablanca (Morocco)
}

\author{
Bienvenu Houssou*, Marième Camara, Romaric Massi, Mohamed Rachid, Abdellah Madani, \\ Meryem Qachouh, Asma Quessar
}

Department of Clinical Hematology and Pediatric Oncology, Hospital August 20, Casablanca, Morocco

\section{Email address:}

houbien85@yahoo.fr(B.Houssou),macodou89@outlook.fr(M.Camara),massiroswork@yahoo.fr(R. Massi), rachid.mohamed.h@gmail.com(M. Rachid),madani.hemato@gmail.com(A. Madani), qachouh.meryem@gmail.com(M. Qachouh), quessar.a@gmail.com(A.Quessar)

${ }^{*}$ Corresponding author

\section{To cite this article:}

Bienvenu Houssou, Marième Camara, Romaric Massi, Mohamed Rachid, Abdellah Madani, Meryem Qachouh, Asma Quessar. Epidemiological, Diagnostic, Therapeutic and Evolutionary Profile of Patients with Follicular Lymphoma from 2012 to 2015 in Casablanca (Morocco). American Journal of Internal Medicine. Vol. 6, No. 3, 2018, pp. 43-46. doi: 10.11648/j.ajim.20180603.11

Received: April 1, 2018; Accepted: April 15, 2018; Published: May 21, 2018

\begin{abstract}
Background: Follicular lymphomas are indolent lymphomas of low incidence in Africa. They are considered incurable, but recent progress has led to a significant increase in survival. The aim of our work is to describe the epidemiological, diagnostic, therapeutic and evolutionary profile of patients followed for follicular lymphoma in Casablanca, Morocco. Patients and methods: During the period of January 1, 2012 to December 31, 2015, were included, all patients diagnosed of follicular lymphoma on a histological and immunohistochemical study of the ganglion or the affected tumor tissue. The treatment was either a therapeutic abstention or chemotherapy with or without Rituximab. Results: 53 (6.4\%) of the 841 patients followed-up for non-Hodgkin's lymphoma had follicular lymphoma, only 35 were included in this study. The median age of diagnosed patients was 56.1 years $[27 ; 87]$ and the sex ratio 2 . The primary site was the ganglion in $28(80 \%)$ patients and $71.5 \%$ of the patients were at stage III and IV of Ann Arbor at diagnosis. According to the FLIPI score 1 about $1 / 3$ of the patients were classified as low risk, intermediate risk and high risk, respectively. $3(9.4 \%)$ patients were under supervision/observation, 1 patient under CVP, 13 (40.6\%) patients on CHOP, 1 patient on R-chloraminophen, 14 (43.6\%) on RCHOP. In the R-CHOP group, $85.7 \%$ of the patients were on CR with a median remission of 25.8 months. 3 patients in the RCHOP group had/received maintenance with rituximab. Discussion and conclusion: Follicular lymphoma is of low frequency and is often revealed by lymphadenopathy. The patients are diagnosed at an advanced stage. The R-CHOP protocol with rituximab maintenance is the most used and best protocol for our patients.
\end{abstract}

Keywords: Follicular Lymphoma, Frequency, Rituximab, Survival

\section{Introduction}

Follicular lymphoma (FL) is a non-Hodgkin lymphoma (NHL) of the B lymphoid lineage derived from germinal center cells: centroblasts and centrocytes $[1,2]$. They represent $70 \%$ of indolent lymphomas [3]. In contrast to developed countries where its incidence is 20 to $25 \%$ of lymphomas $[4,5]$, in developing countries and particularly in Africa it is low: $13.2 \%$ in Algeria, $13.8 \%$ in Egypt [6]. The therapeutic progress of the latter has significantly improved the prognosis with an increase in life expectancy [2].

In Morocco, the management of lymphomas and in particular follicular lymphomas has been improved in recent years, but their incidence is not known due to a lack of registry.

The aim of our work is to describe the epidemiological, clinical, biological and evolutionary profile of patients diagnosed with follicular lymphoma in Casablanca, Morocco. 


\section{Patients and Methods}

During the period of January 1, 2012 to December 31, 2015, were included, all patients diagnosed of follicular lymphoma on a histological and immunohistochemical study of the ganglion or the affected tumor tissue. The panel used for immunophenotyping is CD20, CD10, bc12, bcl6 and CD5. Extension assessment consisted of clinical examination, cervicothoraco-abdomino-pelvic CT scan, chest X-ray, abdominal ultrasound, osteomedullary biopsy, blood count, and LDH assay. The pre-treatment assessment was based on cardiac ultrasound and hepatitis B, C and HIV viral serologies. The prognostic stratification was based on the FLIPI score 1. Only patients who had a high tumor mass were put on treatment. These patients had one of the following criteria: lymph node or extra ganglionic location $>7 \mathrm{~cm}$; at least three lymph node locations in 3 different areas, each with a diameter $>3 \mathrm{~cm}$; troubling localization (pleural or peritoneal effusion, ureteral compression, epidural localization...); splenomegaly beyond the umbilical line; blood cytopenia. The treatment was heterogeneous, either therapeutic abstention or chemotherapy (CHOP (Cyclophosphamide-Adriamycin-Oncovin-Prednisone), CVP (Cyclophosphamide-Vincristine-Prednisone), with or without immunotherapy (R-chloraminophen, R-CHOP) and sometimes a maintenance with Rituximab.

The data were collected retrospectively on a preestablished form containing the epidemiological, diagnostic, extension and pretreatment components. The analysis of the results was by SPSS 20.0 type software. Descriptive statistics were used to summarize the data. The qualitative variables were expressed as frequency, while the quantitative variables were expressed as median and their standard deviations. Correlations between the variables were tested by the chick test. The materiality threshold was set at 0.05 .

\section{Results}

\section{Epidemiological aspects}

During the study period, 841 cases of NHL were identified, $53(6.3 \%)$ of which were of follicular lymphomas. The median age at diagnosis was 56.1 years with extremes ranging from 27 to 87 years. There was a male predominance of 33 men for 20 women with a sex ratio of 1.65.

Of the 18 patients who exercised a professional activity, 10 worked in the agricultural sector, 3 were laborers, 2 in the industrial sector and 3 in the tertiary sector (trade, services).

After the first consultation, we lost sight of 18 patients and only the remaining 35 patients were included for the study of diagnostic, therapeutic and progressive (evolutionary) aspects.

Diagnostic aspects
The median time from the onset of symptoms to the first visit was 9 months with extremes of 2 months to 17 months, and that between the first visit and diagnosis was 9 days with extremes of 1 day to 30 days.

The majority of patients, $22(65.75 \%)$ had a performance status (PS) WHO score between 1 and 2, 4. 11.4\% of the patients had a WHO PS at 0,6.17.1 patients were at a WHO PS at 3 and $3(8.6 \%)$ patients were at WHO PS at 4.

The primary site was the lymph node in $80 \%$ of cases, followed by digestive localization in $8.6 \%$ of cases, the spleen and the bone in $5.7 \%$ of cases each.

According to the classification of Ann Arbor 25 (71.5\%) patients were at stage III and IV and 10 (28.5\%) patients were at stage I and II (table 1).

Table 1. Case Distribution at Diagnosis by Ann Arbor Extension Stage.

\begin{tabular}{lll}
\hline Stratification Ann Arbor & Number of cases & \% \\
\hline I & 4 & 11.4 \\
II & 6 & 17.1 \\
III & 17 & 48.6 \\
IV & 8 & 22.9 \\
Total & 35 & 100 \\
\hline
\end{tabular}

Histologically, the 35 cases of FL were confirmed by anatomy pathology and immunohistochemistry, but only in 20 cases were the histological grades specified, 12 cases, that is to say $60 \%$ were grade 1,7 cases $(35 \%)$ grade 2 and 1 case (5\%) grade $3 \mathrm{~A}$.

The prognostic stratification according to the FLIPI 1 score was established in 32 patients, i.e. approximately $91.4 \%$ and $12(37.5 \%)$ patients were classified as high risk (Table 2).

Table 2. Distribution of cases by FLIPI score 1.

\begin{tabular}{lll}
\hline Prognosticgroup & Number of cases & $\mathbf{\%}$ \\
\hline Low risk & 11 & 34.4 \\
Intermediate risk & 9 & 28.1 \\
High risk & 12 & 37.5 \\
Total & 32 & 100 \\
\hline
\end{tabular}

Therapeutic and evolutionary aspects

The median time from diagnosis to treatment was 4 days with extremes of 0 to 22 days.

Of the 35 patients, $6(17.1 \%)$ were abstaining from therapy, 14 (40\%) had benefited from R-CHOP, 13 (37.1\%) were on CHOP, 1 (2.9\%) on CVP and R-chloraminophen for only one patient.

Of the 14 patients who received R-CHOP, 12 (85.7\%) were in complete remission (CR) with a median $\mathrm{CR}$ duration of 25.8 months, only 3 patients $(21.4 \%)$ received rituximab for maintenance (Table 3).

Table 3. Therapeutic Results.

\begin{tabular}{llllll}
\hline Therapeutic regimen & Number of cases & CR & median CR (mois) & Relapse & Death \\
\hline Abstention & 3 & & & & \\
R-CHOP & 14 & $12(85.7 \%)$ & 25.8 & $2(14.3 \%)$ & 0 \\
CHOP & 13 & $11(84.6 \%)$ & 16.5 & 1 & 0 \\
CVP & 1 & 1 & 26 & 1 & 0 \\
R-chloramin & 1 & 1 & 21 & 1 & 0 \\
\hline
\end{tabular}


Of the 13 patients on CHOP, $11(84.6 \%)$ were on CR with a median CR duration of 16.5 months, and 1 patient was relapsed after 18 months of CR.
The event-free survival patients regardless of the treatment regime at 40 months were $84 \%$ (figure 1).

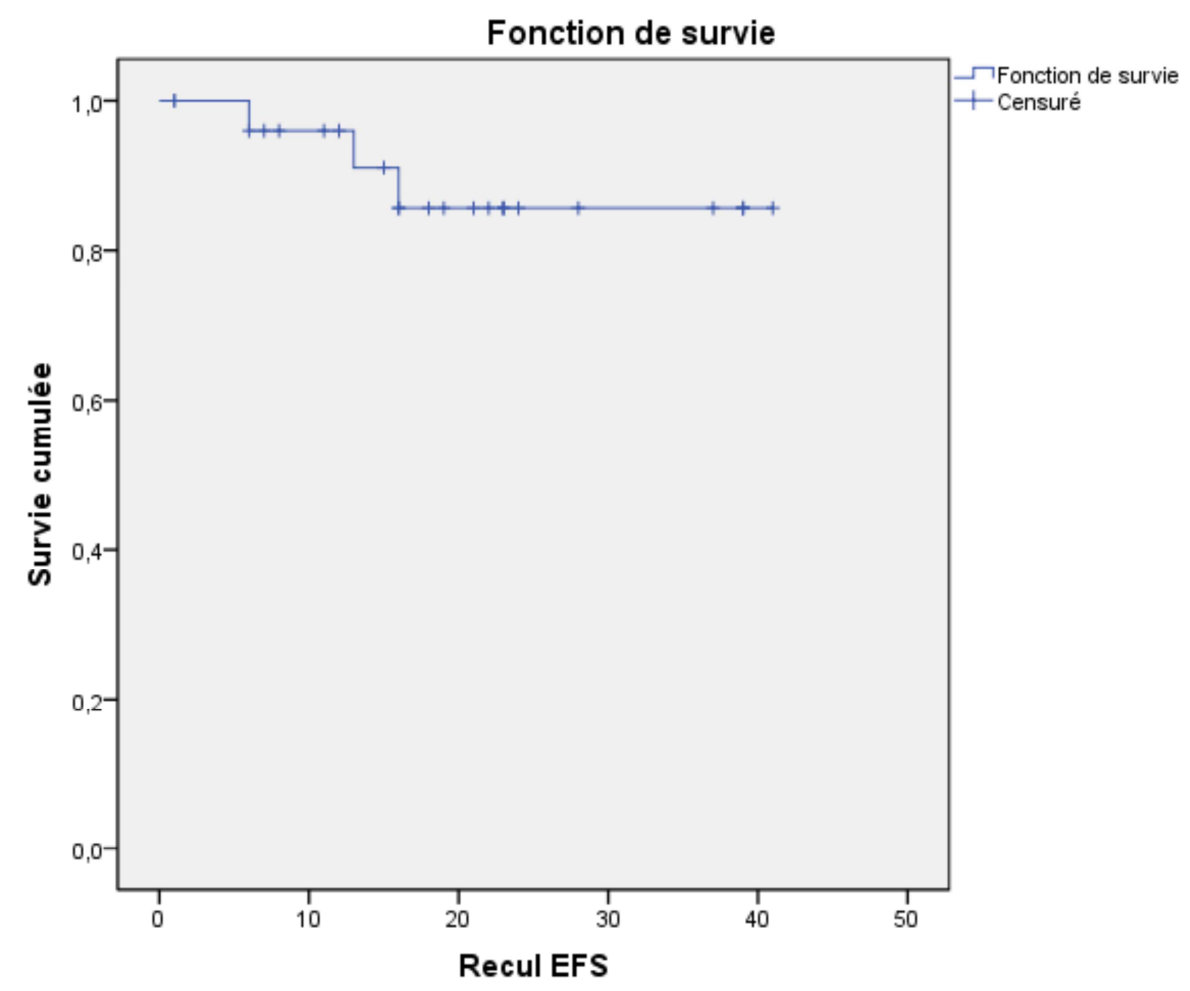

Figure 1. Event-free survival of patients followed for follicular lymphoma.

\section{Discussion}

In our 841 NHL series, FL represents $6.4 \%$. This frequency is that of the largest clinical hematology center in Casablanca, Morocco. There are several other public and private centers for lymphoma management in Morocco; the availability of the registry would permit to have an incidence of lymphoma in general and particularly that of FL. The incidence of FL reported in southern countries is $13.2 \%$ in Algeria, $13.8 \%$ in Egypt [6], $13.3 \%$ in Nigeria [7], 6.3\% in Taiwan [8], 8.1\% in Pakistan [9], 8\% in the Philippines [10] and $1.5 \%$ in Gabon [11]. The incidence is $6.2 \%$ in Korea [8] and $6.4 \%$ in Hong Kong [9]. These different rates contrast with higher rates in the industrialized countries of North America and Western Europe, which vary between 20\% and $33 \%$ [14]. These similarities of frequencies found in Asia and Africa despite their different ethnic origins and geographical locations are more in agreement with a dominant effect of the environment rather than genetic influences. This is also consistent with data derived from Asian emigrants in the United States and their descendants, in which emigrants in the United States appeared to maintain the risk of disease of their country of birth, whereas the incidence of children born in the United States has approached those of whites born in the United States $[15,16]$.

The median age at diagnosis which is 56.1 years is lower than that reported by the team of Sebban C. in 2014, which is
63 years old [17]. The Kane EV team in 2012 found in their study of menstrual and reproductive factors and the use of hormonal contraception in association with non-Hodgkin's lymphoma a slight female predominance sex ratio $\mathrm{H} / \mathrm{F}$ : 1/1.7 in FL [18] contrary to the male predominance found in our study.

The median time between the first consultation and the diagnosis which is 9 days with extremes of 1 day to 30 days is better than the 30 days with the extremes of 8 days to 72 days found by the team of Dakar in 2002 [19]. On the other hand, as in their study, our patients are diagnosed at an advanced stage since $71.5 \%$ are at stage III and IV of Ann Arbor. This large proportion of patients with advanced FL is justified by the delay between the onset of symptoms and the first consultation, which is 2 months to 19 months.

The histological stratification finds grade 1 and 2 in $95 \%$ of cases, which is superimposable to what is reported in the literature [17].

The positron emission tomography (PET) scanner which is now recommended for the initial assessment of extension and evaluation [20, 21] is done in our department only for evaluation of patients in whom the tomodensitometry does not allow a good estimate of the response to therapy.

For the prognostic group classification, the choice of FLIPI 1 was made because not all patients had the $\beta 2$ microglobulin at diagnosis. The low risk group in our study which is $34.4 \%$ is close to the standards which are $36 \%$ [22]. 
The intermediate group that is $28.1 \%$ is less than $37 \%$ reported in the literature [22]. The $37.5 \%$ of the high risk group is more than the standards which are $27 \%$ [22].

The majority of our patients were without social coverage, which makes it difficult to use rituximab because the molecule was expensive; but since 2016 in Morocco, all patients routinely benefit from rituximab. The starting of treatment is based on the presence of criteria of high tumor mass. Patients on R-CHOP have an $85.7 \%$ remission and a median CR duration of approximately 26 months, better than other chemotherapy regimens. It is now the only diet used in our center for FL. Maintenance with rituximab is now a routine.

The survival of patients of all treatment regimens at 40 months is $84 \%$ comparable to international standards [17].

\section{Conclusion}

FL is of low frequency and is often revealed by lymphadenopathy. The patients are diagnosed at an advanced stage. The R-CHOP protocol with rituximab maintenance is the most used and the best protocol for our patients. The establishment of the register will enable us to know the incidence of NHL and particularly that of the FL.

\section{References}

[1] Fisher RI, Leblanc M, Press OW, Maloney DG, Unger JM, Miller TP. New treatement options have changed the survival of patient with follicular lymphoma. J clin Oncol 2005; 23:8447-52.

[2] Sebban C, Brice P, Delarue R, Haioun C, Souleau B, Mounier $\mathrm{N}$, et al. Impact of rituximab and/or high dose therapy with auto transplant at time of relapse in patients with follicular lymphoma: a GELA study. J clin Oncol 2008; 26:3614-20.

[3] Saint M, Allimani C, Tereanu C, De Angelis R, Capocaccia R, Visser $\mathrm{O}$, et al. HEAMACARE working incidence in Europe by morphologic subtype, results oh HAEMACARE project, Blood 2010; 116: 3724-34.

[4] Référentiels SFH. Lymphome folliculaire. Hématologie 2010; 16 (Suppl .4):35-8.

[5] Dreyling M, Ghielmini M, Marcus R et al. ESMO Guidelines Working Group. Newly diagnosed and relapsed follicular lymphoma: ESMO Clinical Practice Guidelines for diagnosis, treatment and follow-up. Ann Oncol 2011;22 (Suppl .6):vi5963.

[6] Perry AM, Diebold J, Nathwani BN, MacLennan KA, MüllerHermelink HK, Bast M, et al. Relative frequency of nonHodgkin lymphoma subtypes in selected centres in North Africa, the middle east and India: a review of 971 cases. Br J Haematol. 2016 Mar; 172(5):699-708.

[7] Obafunwa JO, Akinsete I. Malignant lymphomas in Jos, Nigeria: a ten-year study. Cent Afr J Med. 1992; 38:17-25.

[8] Shih LY, Liang DC. Non-Hodgkin's lymphomas in Asia. Hematol Oncol Clin North Am. 1991; 5:983-1001.

[9] Ahmad M, Khan AH, Mansoor A, Khan MA, Saeed S. NonHodgkin's lymphoma-clinicopathological pattern. J Pak Med
Assoc. 1992; 42: 205-207.

[10] Calderon B, Villalon A, Augustin B, Javier B, Zamuco J, Mori S. Malignant lymphoma in Manila - a clinicopathologic study at the University of the Philippines-Philippine General Hospital Medical Center. Jpn J Clin Oncol. 1986;16:21-27.

[11] Walter PR, Klotz F, Alfy-Gattas T, Minko-Mi-Etoua D, Nguembi-Mbina C. Malignant lymphomas in Gabon (equatorial Africa): a morphologic study of 72 cases. Hum Pathol. 1991; 22:1040-1043.

[12] Ko YH, Kim CW, Park CS, et al. REAL classification of malignant lymphomas in the Republic of Korea: incidence of recently recognized entities and changes in clinicopathologic features: Hematolymphoreticular Study Group of the Korean Society of Pathologists: revised European-American lymphoma. Cancer. 1998;83:806-812.

[13] Ho FC, Todd D, Loke SL, Ng RP, Khoo RK. Clinicopathological features of malignant lymphomas in 294 Hong Kong Chinese patients: retrospective study covering an eightyear period. Int J Cancer. 1984; 34:143-148.

[14] Anderson JR, Armitage JO, Weisenburger DD. Epidemiology of the non-Hodgkin's lymphomas: distributions of the major subtypes differ by geographic locations. Non-Hodgkin's Lymphoma Classification Project. Ann Oncol. 1998;9:717720.

[15] Herrinton LJ, Goldoft M, Schwartz SM, Weiss NS. The incidence of non-Hodgkin's lymphoma and its histologic subtypes in Asian migrants to the United States and their descendants. Cancer Causes Control. 1996; 7:224-230.

[16] James J. Biagi and John F. Seymour. Insights into the molecular pathogenesis of follicular lymphoma arising from analysis of geographic variation. Blood. 2002; 99:4265-4275.

[17] Sebban C, Chassagne Clément C, Nicolas Virrelizier E, Chesquières H. Lymphome folliculaire. EMC-Hématologie: 2014; volume 9, numéro 4: 1-16.

[18] Kane EV, Roman E, Becker N, Bernstein L, Boffetta P, Bracci $\mathrm{PM}$ et al. Menstrual and reproductive factors, and hormonal contraception use: association with non hodgkinien lymphoma in pooled analysis Interlymph case contrôle studies. Ann Oncol 2012; 23: 2362-74

[19] Therapeutic results and evolution of Black African patients with follicular lymphoma: Ivory Coast experience. Koffi G, Kouakou B, Ndiaye FS, Ndathz E, Sanogo I, Sangare A. Bull Cancer. 2007 Oct; 94(10):902-6.

[20] Cheson BD, Pfistner B, Juweid ME, et al. Revised response criteria for malignant lymphoma. J Clin Oncol. 2007; 25(5):579-586.

[21] Stefano Luminari, Sara Galimberti, Annibale Versari, Irene Biasoli, Antonella Anastasia, Chiara Rusconiet al. Positron emission tomography response and minimal residual disease impact on progression-free survival in patients with follicular lymphoma. A subset analysis from the FOLL05 trial of the Fondazione Italiana Linfomi. Haematologica 2016 Feb; 101(2): 66-68.

[22] Solal-Céligny P, Roy P, Colombat P, White J, Armitage JO, Arranz-Saez R, et al. Follicular Lymphoma International Prognostic Index. Blood 2004; 104: 1258-65. 